\title{
THERMODYNAMIC BETHE ANSATZ AND DILOGARITHM IDENTITIES I.
}

\author{
Edward Frenkel and András Szenes
}

\begin{abstract}
We prove an infinite series of multi-parameter dilogarithm identities generalizing the well known Euler and Abel (pentagon) identities. These identities have been recently conjectured by physicists in connection with Thermodynamic Bethe Ansatz.
\end{abstract}

\section{Introduction}

A decade has passed since the seminal work [1] by A. A. Belavin, A. P. Polyakov, and A. B. Zamolodchikov. Since then, much progress has been made in understanding Conformal Field Theories (CFTs) in two dimensions. The success in the study of CFTs is due to their invariance with respect to the Virasoro algebra, or, more generally, extended conformal algebras. This property allows one to describe CFTs in terms of representation theory of infinite dimensional Lie algebras, or vertex operator algebras, and algebraic geometry of complex curves.

In [2] A. B. Zamolodchikov introduced an interesting class of 2D quantum field theories - perturbations of CFTs by relevant operators. These theories lack conformal invariance, but possess some other remarkable algebraic structures, which are yet to be fully understood from the mathematical point of view. One of the properties is the existence of infinitely many local integrals of motion in involution. This was conjectured in [2] (see also [3]) and proved in [4]. Thus, a perturbation of a CFT is an integrable 2D quantum field theory, and as such, it is governed by a purely elastic $S$-matrix, which satisfies various algebraic constraints [5]. These constraints are so strong that knowing the spins of local integrals of motion one can often conjecture the $S$-matrix and hence determine the theory completely, see $[2,6]$ and references therein.

The Thermodynamic Bethe Ansatz (TBA) is a method of verifying these conjectures, which was first applied in this context by Al. B. Zamolodchikov [7]. One starts with an integrable field theory conjectured to be the perturbation of a CFT $\mathcal{T}$, and studies its ultraviolet (UV) behavior. A theory on an infinitely long cylinder of circumference $R$ is described

Received August 23, 1995.

Partially supported by NSF grants. 
by a system of integral equations called the TBA equations. To write down this system explicitly, let us assume that the theory has $N$ species of particles with masses $m_{a}, a=1, \ldots, N$. One is interested in the functions $\epsilon_{a}(\theta)$, which are called the spectral densities of particles of species $a$, see, e.g., [6]. These are functions of the rapidity $\theta$ (recall that rapidity is related to the energy $E$ and the momentum $p$ by the formulas $E=m \cosh \theta, p=m \sinh \theta)$. The TBA equations on the functions $\epsilon_{a}(\theta)$ read

$$
m_{a} R \cosh \theta=\epsilon_{a}(\theta)+\frac{1}{2 \pi} \sum_{b=1}^{N} \int_{-\infty}^{\infty} d \theta^{\prime} \phi_{a b}\left(\theta-\theta^{\prime}\right) \log \left(1+Y_{a}\left(\theta^{\prime}\right)\right),
$$

where $Y_{a}(\theta)=e^{-\epsilon_{a}(\theta)}, \phi_{a b}(\theta)=-i \frac{\partial \log S_{a b}}{\partial \theta}$, and $S_{a b}(\theta)$ is the $S$-matrix.

The TBA equations are usually hard to solve, but one can extract a lot of information from them even without solving them explicitly. The ground state energy of the theory is given by

$$
E(R)=-\frac{1}{2 \pi} \sum_{a=0}^{N} \int_{-\infty}^{\infty} d \theta m_{a} R \cosh \theta \log \left(1+Y_{a}(\theta)\right) .
$$

In the UV limit $R \rightarrow 0$, in which one is supposed to recover the initial CFT $\mathcal{T}$, we should have $E(R) \simeq-\pi \widetilde{c}(R) / 6 R$, where $\widetilde{c}(R) \sim \widetilde{c}+O(R)$. From (1.2) one finds using the TBA equations, see, e.g., [6],

$$
\frac{\pi^{2}}{6} \widetilde{c}=\sum_{a=1}^{N} L\left(\frac{1}{1+y_{a}}\right)
$$

where $L(z)$ is the Rogers dilogarithm function [8]

$$
L(z)=\frac{1}{2} \int_{0}^{z}(\log w d \log (1-w)-\log (1-w) d w), \quad 0 \leq z \leq 1
$$

and $y_{a}=\lim _{R \rightarrow 0} Y_{a}(\theta)$. The numbers $y_{a}$ satisfy the system of algebraic equations

$$
y_{a}=\prod_{b=1}^{N}\left(1+\frac{1}{y_{b}}\right)^{N_{a b}}
$$

where $N_{a b}$ is the number of poles of $S_{a b}(\theta)$ in the upper half plane; in particular, they do not depend on $\theta$.

If the conjectural description of the perturbation of the CFT $\mathcal{T}$ is correct, the number $\widetilde{c}$ in the left-hand side of the formula (1.3) should coincide 
with the effective central charge of $\mathcal{T}$. But in that case formula (1.3) can be considered as a dilogarithm identity, which relates a rational number $\widetilde{c}$ to the algebraic numbers $y_{a}$ 's.

Many dilogarithm identities have been discovered this way in recent years. While the TBA method has not yet been made rigorous, the identities have been proved rigorously by other methods, see $[8,9,10]$. Mathematically, the dilogarithm identities manifest a connection between 2D quantum field theory on the one hand and algebraic $K$-theory and number theory on the other, see [10]. We hope that better understanding of the TBA will enable us to gain new insights into this connection.

Recently, F. Gliozzi and R. Tateo [11] made an important step in this direction. They found functional analogues of the identities (1.3) for a large class of theories, which are labeled by pairs $(G, H)$ of Dynkin diagrams of types $A D E$ and $T$ (the latter is the diagram of type $A$ with a loop attached to one of the end vertices). In such a theory, the species of particles are labeled by pairs of indices $a=1, \ldots, r_{G}$, and $b=1, \ldots, r_{H}$, where $r_{G}$ and $r_{H}$ are the numbers of vertices in the diagrams $G$ and $H$, respectively.

The main fact, which is due to Al. B. Zamolodchikov [12] and, in the general case, to F. Ravanini, A. Valleriani and R. Tateo [13] is that any solution $\left\{Y_{a}^{b}(\theta)\right\}$ of the TBA equations (1.1) corresponding to the $(G, H)$ theory satisfies the following system of algebraic equations:

$$
Y_{a}^{b}\left(\theta+\frac{\pi I}{h_{G}^{\vee}}\right) Y_{a}^{b}\left(\theta-\frac{\pi I}{h_{G}^{\vee}}\right)=\prod_{c=1}^{r_{G}}\left(1+Y_{c}^{b}(\theta)\right)^{G_{a c}} \prod_{d=1}^{r_{H}}\left(1+\frac{1}{Y_{a}^{d}(\theta)}\right)^{-H_{b d}},
$$

where $I=\sqrt{-1},\left(G_{a c}\right)$ and $\left(H_{b d}\right)$ are the adjacency matrices of the diagrams $G$ and $H$, respectively, and $h_{G}^{\vee}$ is the dual Coxeter number of $G$.

The $Y$-system (1.6) and the closely related $T$-system play an important role in quantum field theory and statistical mechanics $[13,14,15,16]$. In [17] it was conjectured that a certain class of solutions of this system parametrizes the spectra of commuting integrals of motion acting on minimal representations of conformal algebras.

Al. B. Zamolodchikov [12] has conjectured an important periodicity property of solutions of the system (1.6):

$$
Y_{a}^{b}\left(\theta+\pi I \frac{h_{G}^{\vee}+h_{H}^{\vee}}{h_{G}^{\vee}}\right)=Y_{\bar{a}}^{\bar{b}}(\theta)
$$

where $h_{H}^{\vee}$ is the dual Coxeter number of $H$, and $\bar{a}, \bar{b}$ are the vertices conjugate to $a, b$, respectively. This periodicity property allows one to find the conformal dimension of the field responsible for the perturbation of the corresponding CFT, see [12]. 
Now we can write down the dilogarithm identities conjectured in [11]. Let $\left\{Y_{a}^{b}(\theta)\right\}$ be a solution of the $Y$-system (1.6). Fix $\theta$ and set

$$
X_{a}^{b}(m)=\frac{Y_{a}^{b}\left(\theta+\pi I m / h_{G}^{\vee}\right)}{1+Y_{a}^{b}\left(\theta+\pi I m / h_{G}^{\vee}\right)} .
$$

Suppose that all $X_{a}^{b}(m)$ are real numbers between 0 and 1 . Then

$$
\sum_{a=1}^{r_{G}} \sum_{b=1}^{r_{H}} \sum_{m=1}^{h_{G}^{\vee}+h_{H}^{\vee}} L\left(X_{a}^{b}(m)\right)=\frac{\pi^{2}}{6} r_{G} r_{H} h_{G}^{\vee} .
$$

Let $y_{a}^{b}=\lim _{\theta \rightarrow+\infty} Y_{a}^{b}(\theta)$. In the limit $\theta \rightarrow+\infty$ the system (1.6) becomes a system of type (1.5), and the identity (1.8) becomes equivalent to the identity (1.3) corresponding to the UV limit of the $(G, H)$ theory (in order to relate them, one has to use the Euler identity $\left.L(z)+L(1-z)=\pi^{2} / 6\right)$. Therefore, the identities (1.8) can be viewed as functional analogues of the known dilogarithm identities (1.3). It is interesting that the identity corresponding to the $\left(A_{1}, A_{1}\right)$ theory is the Euler identity above, and the identity corresponding to the $\left(A_{2}, A_{1}\right)$ theory is the pentagon identity of the dilogarithm function, see [11].

There are many indications that analogues of the $Y$-system can be defined for other integrable field theories and that there are dilogarithm identities associated to them, see [18].

In this paper, we give a proof of the periodicity conjecture (1.7) and the identities (1.8) and their generalizations for the $\left(A_{n}, A_{1}\right)$ theories. We also prove analogous identities for the Bloch-Wigner function, which is the imaginary counterpart of the Rogers dilogarithm. Our proof of these identities relies on a universal property of the dilogarithm functions, which for the Bloch-Wigner function was first proved by S. Bloch [19].

Our approach can be generalized to the identities corresponding to more general diagrams. We have already obtained a complete proof of periodicity and dilogarithm identities of $\left(A_{n}, A_{2}\right)$-type and partial results in the general case. We will report on those results in the second part of this paper.

Upon completing this work, we learned about the paper [20], in which another approach to the dilogarithm identities (1.8) was proposed and a proof, different from ours, of the periodicity (1.7) and the identities (1.8) for the $\left(A_{n}, A_{1}\right)$ theories was outlined.

The paper is organized as follows. In Section 2 we prove the periodicity property of the $Y$-system. In Section 3 we give a general form of the dilogarithm identities for the Rogers and the Bloch-Wigner dilogarithms. 
In Section 4 we prove the identities (1.8) of $\left(A_{n}, A_{1}\right)$-type and their generalizations.

\section{Periodicity}

In the case of $\left(A_{n}, A_{1}\right)$ theory the $Y$-system (1.6) takes the form

$$
Y_{a}\left(\theta+\frac{\pi I}{n+1}\right) Y_{a}\left(\theta-\frac{\pi I}{n+1}\right)=\left(1+Y_{a-1}(\theta)\right)\left(1+Y_{a+1}(\theta)\right)
$$

where we put $Y_{a}(\theta)=Y_{a}^{1}(\theta)$.

In this section we prove the periodicity property of this system.

Let us fix $\theta$ and denote

$$
Y(i, j)=Y_{j}\left(\theta+\pi I \frac{i}{n+1}\right), i \in \mathbb{Z}, j=1, \ldots, n .
$$

In this notation the system (2.1) reads

$$
Y(i-1, j) Y(i+1, j)=(1+Y(i, j-1))(1+Y(i, j+1)),
$$

$i \in \mathbb{Z}, j=1, \ldots, n$, where we put $Y(i, 0)=Y(i, n+1)=0$ for all $i$.

Remark. In this paper we treat the system (2.2) as a system of algebraic equations on the variables $Y(i, j)$. We do not restrict ourselves to the special case when $Y(i, j)$ are values of functions $Y_{j}(\theta)$ satisfying the $Y$ system (2.1) and therefore we do not use any global properties of functional solutions $Y_{j}(\theta)$ of $(2.1)$.

Theorem 1. Suppose that the variables $Y(i, j), i \in \mathbb{Z}, j=1, \ldots, n$, satisfy the system of equations (2.2). Then $Y(i, j)=Y(i+n+3, n+1-j)$.

Proof. It will be helpful to pass to the new variables $X(i, j)=Y(i, j) /(1+$ $Y(i, j))$. In these, $(2.2)$ takes the form

$$
\begin{aligned}
\left(1-\frac{1}{X(i-1, j)}\right) & \left(1-\frac{1}{X(i+1, j)}\right) \\
& =(1-X(i, j-1))(1-X(i, j+1)) .
\end{aligned}
$$

Also introduce the transformation $S:(i, j) \rightarrow(i+n+3, n+1-j)$. Thus our goal is to show that $Y(i, j)=Y(S(i, j))$.

The method of proof is setting up some initial conditions and then solving the system of equations explicitly. Note that the variables $Y(i, j)$ and $X(i, j)$ with $i+j$ even are independent from those with $i+j$ odd. Therefore, without loss of generality, we can restrict ourselves to those $X(i, j)$ for which $i+j$ is even.

Introduce a set of variables $a_{1}, a_{2}, \ldots, a_{n}$, and set $X(i, i)=a_{i}$ for $i=$ $1, \ldots n$. The condition $Y(i, 0)=Y(i, n+1)=0$ translates into $a_{0}=a_{n+1}=$ 
0 . It is clear that these initial conditions together with (2.3) determine all the $X(i, j)$ 's. Moreover, the relations of (2.3) impose no relations on the $a_{i}$ 's. Therefore, $a_{1}, \ldots, a_{n}$ can be considered as parameters of the solutions of the system (2.3).

By successively applying (2.3), we can express the variables $X(i+2, i)$, $i=1, \ldots, n$ in terms of the $a_{i}$ 's. The result is surprisingly simple:

$$
X(i+2, i)=\frac{1-a_{1} a_{2} \cdots a_{i}}{1-a_{1} a_{2} \cdots a_{i+1}} .
$$

To prove this formula, it is enough to check that relations (2.3) hold identically if we substitute (2.4) into them. To this end, consider (2.3) for $i=j+1$. Substitute the above expression for $X(j+1, j-1)$ and $X(j+2, j)$, and also $a_{j}$ and $a_{j+1}$ for $X(j, j)$ and $X(j+1, j+1)$, respectively. After simple manipulations we obtain

$$
\frac{1-a_{j}}{a_{j}} \cdot \frac{a_{1} a_{2} \cdots a_{j}\left(1-a_{j+1}\right)}{1-a_{1} a_{2} \cdots a_{j}}=\left(a_{j+1}-1\right) \frac{a_{1} a_{2} \cdots a_{j-1}\left(a_{j}-1\right)}{1-a_{1} a_{2} \cdots a_{j}}
$$

which is an obvious identity. This proves formula (2.4).

An important corollary of (2.4) is that

$$
X(n+2, n)=1-a_{1} a_{2} \cdots a_{n},
$$

since $a_{n+1}=0$ by definition.

Now observe that we can apply the same recursion "starting at the other end", i.e., do the calculations symmetrically with respect to the point $((n+1) / 2,(n+1) / 2)$. Then we obtain an expression for $X(n-i-1, n-i+1)$, which is equal to the expression for $X(i+2, i)$ with $a_{i}$ replaced by $a_{n+1-i}$ :

$$
X(n-i-1, n-i+1)=\frac{1-a_{n} a_{n-1} \cdots a_{n+1-i}}{1-a_{n} a_{n-1} \cdots a_{n-i}} .
$$

In particular, when $i=n$ we have

$$
X(-1,1)=1-a_{n} a_{n-1} \cdots a_{1}
$$

and thus $X(n+2, n)=X(-1,1)$ or $X(S(-1,1))=X(-1,1)$ (see the picture below in the case $n=4)$. 


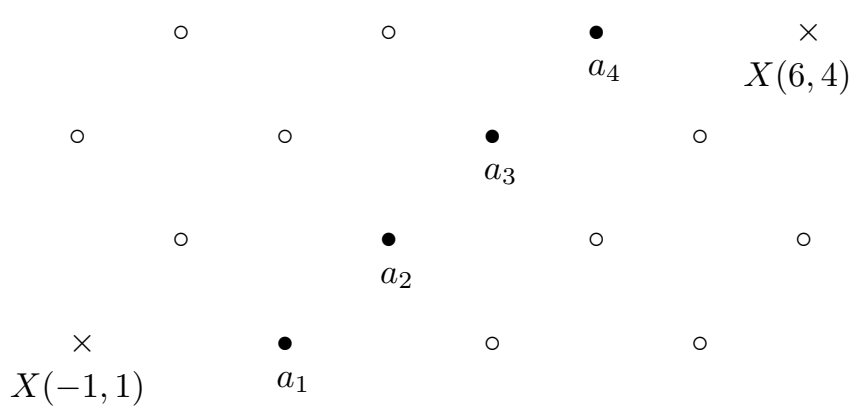

Note that the key fact is that formula $(2.5)$ for $X(n+2, n)$ is invariant with respect to the involution $a_{i} \rightarrow a_{n+1-i}$.

Since the system (2.3) is invariant with respect to simultaneous shifts of the coordinate $i$, we conclude that $X(i, 1)=X(S(i, 1))$ for all $i \in \mathbb{Z}$, i.e., the periodicity holds for $X(i, 1), i \in \mathbb{Z}$. Clearly, these variables determine all the others via (2.3). Now note that (2.3) is invariant under $S$, thus the periodicity holds for all of the $X(i, j)$ 's and hence for all the $Y(i, j)$ 's.

\section{Properties of the dilogarithm functions}

We consider two different types of dilogarithm functions: the Rogers dilogarithm and the Bloch-Wigner dilogarithm. In this section, we discuss a universal property of these functions, which will allow us to prove the dilogarithm identities (1.8) and their generalizations. In the case of the Bloch-Wigner function, this property was first proved by S. Bloch [19].

First let us introduce some notation.

For an abelian group $A$, consider the additive abelian group $A \otimes_{\mathbb{Z}} A$. It consists of finite sums

$$
\sum_{i} n_{i} \cdot g_{i} \otimes h_{i}, \quad g_{i}, h_{i} \in A, n_{i} \in \mathbb{Z},
$$

with the obvious addition, and subject to the relations

$$
\begin{gathered}
(f g) \otimes h=f \otimes h+g \otimes h, \quad h \otimes(f g)=h \otimes f+h \otimes g, \\
1 \otimes h=h \otimes 1=0, \\
f^{-1} \otimes g=-f \otimes g, \quad g \otimes f^{-1}=-g \otimes f .
\end{gathered}
$$

Denote by $S^{2} A$ the subgroup of $A \otimes_{\mathbb{Z}} A$ generated by elements of the form $a \otimes b+b \otimes a$ for all $a, b \in A$. 
3.1. The first identity for the Rogers dilogarithm. Let $C$ be the multiplicative group of nowhere vanishing continuous differentiable functions from $[0,1]$ to $\mathbb{R}_{+}=(0,+\infty)$. Denote by $L(z)$ the Rogers dilogarithm function defined on the interval $[0,1]$ by formula (1.4).

Proposition 1. Let $f_{1}, \ldots, f_{N}$ be continuous differentiable functions from $[0,1]$ to $(0,1)$, such that

$$
\sum_{i=1}^{N} f_{i} \otimes\left(1-f_{i}\right) \in S^{2} C
$$

Then

$$
\sum_{i=1}^{N} L\left(f_{i}(x)\right)=\mathrm{const}
$$

as a function of $x \in[0,1]$.

Proof. By the definition of the Rogers dilogarithm function (1.4), we have, for $0<z<1$,

$$
d L(z)=\frac{1}{2}(\log z \cdot d \log (1-z)-\log (1-z) \cdot d \log z)
$$

which implies that

$$
\begin{aligned}
& d \sum_{i=1}^{N} L\left(f_{i}(x)\right) \\
& =\frac{1}{2} \sum_{i=1}^{N}\left(\log f_{i}(x) \cdot d \log \left(1-f_{i}(x)\right)-\log \left(1-f_{i}(x)\right) \cdot d \log f_{i}(x)\right) .
\end{aligned}
$$

If the $f_{i}$ 's satisfy the condition of the proposition, then there exist $g_{j}, h_{j} \in C, j=1, \ldots, m$, such that

$$
\sum_{i=1}^{N} f_{i} \otimes\left(1-f_{i}\right)=\sum_{j=1}^{m}\left(g_{j} \otimes h_{j}+h_{j} \otimes g_{j}\right)
$$

For $x, y \in[0,1]$ we can define a homomorphism $\log _{x, y}: C \otimes C \rightarrow \mathbb{R}$, where $\mathbb{R}$ is considered as an additive group, by the formula $\log _{x, y}(f \otimes g)=$ $\log f(x) \cdot \log g(y)$. By applying the homomorphism $\log _{x, y}$ to both sides of formula (3.3), we obtain

$$
\sum_{i=1}^{N} \log f_{i}(x) \log \left(1-f_{i}(y)\right)=\sum_{j=1}^{m} \log g_{j}(x) \log h_{j}(y)+\log h_{j}(x) \log g_{j}(y) .
$$


Hence

$$
\begin{aligned}
\sum_{i=1}^{N} d \log f_{i}(x) & \cdot \log \left(1-f_{i}(y)\right) \\
& =\sum_{j=1}^{m} d \log g_{j}(x) \cdot \log h_{j}(y)+d \log h_{j}(x) \cdot \log g_{j}(y)
\end{aligned}
$$

and

$$
\begin{aligned}
\sum_{i=1}^{N} \log f_{i}(x) \cdot & d \log \left(1-f_{i}(y)\right) \\
= & \sum_{j=1}^{m} \log g_{j}(x) \cdot d \log h_{j}(y)+\log h_{j}(x) \cdot d \log g_{j}(y) .
\end{aligned}
$$

Subtracting (3.4) from (3.5) and setting $y=x$, we obtain

$$
\sum_{i=1}^{N}\left(\log f_{i}(x) \cdot d \log \left(1-f_{i}(x)\right)-\log \left(1-f_{i}(x)\right) \cdot d \log f_{i}(x)\right)=0,
$$

which implies by (3.2) that

$$
d \sum_{i=1}^{N} L\left(f_{i}(x)\right)=0 \text {. }
$$

Corollary 1. Let $f_{1}, \ldots, f_{N}$ be as in Proposition 1. Then

$$
\sum_{i=1}^{N} L\left(f_{i}(0)\right)=\sum_{i=1}^{N} L\left(f_{i}(1)\right)
$$

3.2. The second identity for the Rogers function. The Rogers dilogarithm function can be extended to the whole real axis as follows. Set

$$
L(z)=\frac{\pi^{2}}{3}-L\left(\frac{1}{z}\right), \quad z>1
$$

and

$$
L(z)=-L\left(\frac{z}{z-1}\right), \quad z<0 .
$$

This is a continuous function on $\mathbb{R}$, which is differentiable for all $z$ except 0 and 1 . It follows from this definition that

$$
\lim _{z \rightarrow+\infty} L(z)=\frac{\pi^{2}}{3}, \quad \lim _{z \rightarrow-\infty}=-\frac{\pi^{2}}{6} .
$$


These limits differ by $\pi^{2} / 2$, and hence $L(z)$ can be extended to $\infty$ as a function with values in $\mathbb{R} /\left(\pi^{2} / 2\right) \mathbb{Z}$. This way we obtain a function from $\mathbb{R P}^{1}=\mathbb{R} \cup\{\infty\}$ to $R /\left(\pi^{2} / 2\right) \mathbb{Z}$, which we denote by $\mathcal{L}(z)$. The function $\mathcal{L}(z)$ is continuous and differentiable for all $z$ except 0 and 1 .

Now let $\mathcal{C}$ be the multiplicative group of nonzero rational functions $[0,1] \rightarrow \mathbb{R}$.

Proposition 2. Suppose that $f_{1}, \ldots, f_{N} \in \mathcal{C}$ are non-constant functions, such that

$$
\sum_{i=1}^{N} f_{i} \otimes\left(1-f_{i}\right) \in S^{2} \mathcal{C}
$$

Then

$$
\sum_{i=1}^{N} \mathcal{L}\left(f_{i}(x)\right)=\mathrm{const}
$$

as a function of $x \in[0,1]$.

Proof. The proof goes along the lines of the proof of Proposition 1. We consider each $f_{i}(x)$ as a continuous function $[0,1] \rightarrow \mathbb{R P}^{1}$. For generic $x \in[0,1]$, the value $f_{i}(x)$ lies in one of the intervals $(0,1),(1,+\infty)$, or $(-\infty, 0)$. Therefore, the function $\sum_{i=1}^{N} \mathcal{L}\left(f_{i}(x)\right)$ is differentiable for generic $x$. We want to show that its differential vanishes.

Let us show that if $f_{i}(y)$ belongs to one of the intervals, then

$2 d L\left(f_{i}(y)\right)=\log \left|f_{i}(y)\right| \cdot d \log \left|1-f_{i}(y)\right|-\log \left|1-f_{i}(y)\right| \cdot d \log \left|f_{i}(y)\right|$

We consider the three cases separately. If $f_{i}(y) \in(0,1)$ then this follows from formula $(3.1)$.

If $f_{i}(y) \in(1,+\infty)$, we have, by formulas (3.6) and (3.1),

$$
\begin{aligned}
& 2 d L\left(f_{i}(y)\right)=-2 d L\left(\frac{1}{f_{i}(y)}\right) \\
& =\log \left(\frac{1}{f_{i}(y)}\right) \cdot d \log \left(1-\frac{1}{f_{i}(y)}\right)-\log \left(1-\frac{1}{f_{i}(y)}\right) \cdot d \log \left(\frac{1}{f_{i}(y)}\right) \\
& =\log \left|f_{i}(y)\right| \cdot d \log \left|1-f_{i}(y)\right|-\log \left|1-f_{i}(y)\right| \cdot d \log \left|f_{i}(y)\right|
\end{aligned}
$$


If $f_{i}(y) \in(-\infty, 0)$, we have, by formulas (3.7) and (3.1),

$$
\begin{aligned}
2 d L\left(f_{i}(y)\right) & =-2 d L\left(\frac{f_{i}(y)}{f_{i}(y)-1}\right) \\
= & \log \left(\frac{f_{i}(y)}{f_{i}(y)-1}\right) \cdot d \log \left(1-\frac{f_{i}(y)}{f_{i}(y)-1}\right) \\
& \quad-\log \left(1-\frac{f_{i}(y)}{f_{i}(y)-1}\right) \cdot d \log \left(\frac{f_{i}(y)}{f_{i}(y)-1}\right) \\
= & \log \left|f_{i}(y)\right| \cdot d \log \left|1-f_{i}(y)\right|-\log \left|1-f_{i}(y)\right| \cdot d \log \left|f_{i}(y)\right| .
\end{aligned}
$$

Now for $x \in[0,1]$ let $\mathcal{C}_{x}$ be the subgroup of $\mathcal{C}$ which consists of those functions which have neither a zero or pole at $x$. Define for $x, y \in[0,1]$ a homomorphism $\mathcal{L}_{\log }{ }_{x, y}: \mathcal{C}_{x} \otimes \mathcal{C}_{y} \rightarrow \mathbb{R}$ by the formula $\mathcal{L}_{o g} g_{x, y}(f \otimes g)=$ $\log |f(x)| \cdot \log |g(y)|$. Using this homomorphism and the formulas above in the same way as in the proof of Proposition 1, we obtain that

$$
d \sum_{i=1}^{N} L\left(f_{i}(x)\right)=0 \quad \text { for generic } x \in[0,1] .
$$

Therefore, by continuity, $\sum_{i=1}^{N} \mathcal{L}\left(f_{i}(x)\right)=$ const for all $x \in[0,1]$.

Corollary 2. Let $f_{1}, \ldots, f_{N}$ be as in Proposition 2. Then

$$
\sum_{i=1}^{N} L\left(f_{i}(0)\right)=\sum_{i=1}^{N} L\left(f_{i}(1)\right) \quad \bmod \frac{\pi^{2}}{2} .
$$

3.3. Identity for the Bloch-Wigner function. $D: \mathbb{C} \rightarrow \mathbb{R}$, the function given by the formula

$$
D(z)=-\operatorname{Im} \int_{0}^{z} \log (1-w) d \log w+\log |z| \operatorname{Arg}(1-z)
$$

is the Bloch-Wigner function. This function is single-valued and real analytic everywhere except for $z=0,1$, where it is only continuous [19].

Let $\mathfrak{C}$ be the multiplicative group of non-vanishing holomorphic functions $\mathcal{D}_{R} \rightarrow \mathbb{C}$, where $\mathcal{D}_{R}$ is the disc of radius $R>1$.

Proposition 3 ([19]). Suppose that $f_{1}, \ldots, f_{N}$ are holomorphic functions such that $f_{i}, 1-f_{i} \in \mathfrak{C}$ for all $i=1, \ldots, N$, and that

$$
\sum_{i=1}^{N} f_{i} \otimes\left(1-f_{i}\right) \in S^{2} \mathfrak{C}
$$


Then

$$
\sum_{i=1}^{N} D\left(f_{i}(x)\right)=\text { const }
$$

as a function of $x \in \mathcal{D}_{R}$.

The proof is similar to the proofs of the previous identities. Let us observe that

$$
d D(z)=\log |z| d \operatorname{Arg}(1-z)-\log |1-z| d \operatorname{Arg} z .
$$

To prove the proposition we should consider the homomorphism

$$
\mathfrak{L} \mathfrak{o} \mathfrak{g}_{x, y}: \mathfrak{C} \otimes \mathfrak{C} \rightarrow \mathbb{R} \otimes(\mathbb{R} / 2 \pi)
$$

given by $\mathfrak{L o g}_{x, y}(f \otimes g)=\log |f(x)| \otimes \operatorname{Arg} g(y)$ and proceed in the same way as above (see also [19]).

Corollary 3. Let $f_{1}, \ldots, f_{N}$ be as in Proposition 3 . Then

$$
\sum_{i=1}^{N} D\left(f_{i}(0)\right)=\sum_{i=1}^{N} D\left(f_{i}(1)\right)
$$

\section{The dilogarithm identities}

In this section we prove the following result.

\section{Theorem 2.}

(1) Suppose that the real numbers $X(i, j), i \in \mathbb{Z}, j=1, \ldots, n$, satisfy the system of equations (2.3). If all $X(i, j) \in(0,1)$, then

$$
\sum_{i=1}^{n+3} \sum_{j=1}^{n} L(X(i, j))=\frac{\pi^{2}}{6} n(n+1),
$$

and, in general,

$$
\sum_{i=1}^{n+3} \sum_{j=1}^{n} L(X(i, j))=\frac{\pi^{2}}{6} n(n+1) \quad \bmod \frac{\pi^{2}}{2} .
$$

(2) Suppose that the complex numbers $X(i, j), j \in \mathbb{Z}, i=1, \ldots, n$, satisfy the system of equations (2.3). Then

$$
\sum_{i=1}^{n+3} \sum_{j=1}^{n} D(X(i, j))=0 .
$$


First we prove a generalization of (2.5). For $\epsilon=0,1$ let

$$
\mathfrak{S}_{\epsilon}=\{(i, j) \mid i \in \mathbb{Z}, 1 \leq j \leq n, i+j=\epsilon \bmod 2\} .
$$

Let $\mathfrak{F}_{\epsilon}$ be the coset $\mathfrak{S}_{\epsilon} /\{S(i, j) \sim(i, j)\}$, where $S$ is the transformation introduced in $\S 2$, and $p_{\epsilon}: \mathfrak{S}_{\epsilon} \rightarrow \mathfrak{F}_{\epsilon}$ be the corresponding projection. According to Theorem 1 , if the $X(i, j)$ 's satisfy the system $(2.3)$, then $X(i, j)=X(S(i, j))$. Therefore, the $X(i, j)$ 's can be considered a function on $\mathfrak{F}_{0} \cup \mathfrak{F}_{1}$.

For $(i, j) \in \mathfrak{S}_{\epsilon}$, introduce the cone $\mathfrak{C}_{\epsilon}(i, j) \subset \mathfrak{F}_{\epsilon}$ as the image of the set

$$
\left\{\left(i^{\prime}, j^{\prime}\right) \in \mathfrak{S}_{\epsilon}|| i^{\prime}-i|\geq| j^{\prime}-j \mid\right\}
$$

under the map $p_{\epsilon}$. We note that the restriction of $p_{\epsilon}$ to $\mathfrak{C}_{\epsilon}(i, j)$ is injective. Finally, denote by $\mathfrak{B}_{\epsilon}(i, j)=\mathfrak{F}_{\epsilon} \backslash \mathfrak{C}_{\epsilon}(i, j)$ the cone's complement.

Proposition 4. For any $(i, j) \in \mathfrak{F}_{\epsilon}$,

$$
1-X(i, j)=\prod_{\left(i^{\prime}, j^{\prime}\right) \in \mathfrak{B}_{\epsilon}(i, j)} X\left(i^{\prime}, j^{\prime}\right)
$$

Proof. We prove the proposition by induction on $j$. For $j=1$, the set $\mathfrak{B}_{\epsilon}(i, 1)$ is the image of the set $\left\{(i+l+1, l) \in \mathfrak{S}_{\epsilon}\right\}$ under the map $p_{\epsilon}$, and (4.4) is equivalent to (2.6).

Assume now that the proposition is true for all $j<k$. For a fixed $i$ the relation (2.3) between $X(i-1, k-1), X(i+1, k-1), X(i, k-2)$ and $X(i, k)$ gives

$$
1-X(i, k)=\frac{1}{1-X(i, k-2)} \cdot \frac{X(i-1, k-1)}{1-X(i-1, k-1)} \cdot \frac{X(i+1, k-1)}{1-X(i+1, k-1)} .
$$

By our inductive assumption, we can make the substitution (4.4) for the factors of the form $1-X$ in the right hand side. Then we see that the validity of formula (4.4) for $X(i, k)$ is equivalent to the statement

$$
\begin{aligned}
\mathfrak{B}(i-1, k-1)+ & \mathfrak{B}(i+1, k-1) \\
= & \mathfrak{B}(i, k-2)+\mathfrak{B}(i, k)+\{(i-1, k-1),(i+1, k-1)\},
\end{aligned}
$$

where by addition of sets we mean the union of their elements counted with multiplicities. This last statement is a simple fact of elementary geometry.

Note that at the two ends, when $k=1$ or $n+1$, one of the factors in (4.5) is missing. This corresponds to the fact that $\mathfrak{C}(i, 0)=\mathfrak{C}(i, n+1)=\mathfrak{F}_{n}$ and hence $\mathfrak{B}_{\epsilon}(i, 0)=\mathfrak{B}_{\epsilon}(i, n+1)=\emptyset$. 
Proof of Theorem 2. We show that any solution of (2.3) can be connected to a particular solution $X(i, j)_{0}$ for which the sum of values of the dilogarithm functions is known.

The solution $X(i, j)_{0}$ corresponds to the UV limit, i.e., $X(i, j)_{0}$ is $i$ independent. Therefore, the system (2.3) becomes

$$
X(i, j)^{2}=\prod_{l=1}^{n}(1-X(i, j))^{A_{j l}}
$$

where $\left(A_{i j}\right)$ is the Cartan matrix of type $A_{n}$. The following formula gives a particular solution of this system:

$$
X(i, j)_{0}=1-\frac{\sin ^{2} \frac{\pi}{n+3}}{\sin ^{2} \frac{\pi(i+1)}{n+3}}, \quad i \in \mathbb{Z} ; j=1, \ldots, n
$$

(see, e.g., [10]).

Solutions of the system (2.3) are in one-to-one correspondence with the numbers $X(0, j)$ and $X(1, j)$, where $j=1, \ldots, n$. We can choose them arbitrarily, and if none of them is equal to 0 or 1 , then all other numbers $X(i, j)$ can be uniquely determined recursively using the system $(2.3)$.

From now on we restrict our attention to the identity (4.1). The identities (4.2) and (4.3) can be treated in the same fashion.

We have $X(i, j)_{0} \in(0,1)$. Let $\left\{X(i, j)_{1}\right\}$ be another solution of $(2.3)$ such that all $X(i, j)_{1} \in(0,1)$. Consider the functions

$$
X(i, j)(z)=z X(i, j)_{1}+(1-z) X(i, j)_{0}, \quad i=0,1 ; \quad j=1, \ldots, n .
$$

Using these functions, we can determine all other functions $X(i, j)(z)$ uniquely by using recursion from the system (2.3). The functions $X(i, j)(z)$ constructed this way satisfy the system (2.3) for all $z \in[0,1]$ and

$$
X(i, j)(0)=X(i, j)_{0}, X(i, j)(1)=X(i, j)_{1} \text { for all } i, j .
$$

Lemma 1. Let $X(i, j)=X(i, j)(z), i \in \mathbb{Z}, j=1, \ldots, n$, be functions $[0,1] \rightarrow \mathbb{R}$, which satisfy the system $(2.3)$ for all $z \in[0,1]$. Suppose that

(1) $0<X(i, j)(0)<1$ for all $i, j$;

(2) $X(i, j)(z), i=0,1$, are continuous differentiable functions;

(3) $0<X(i, j)(z)<1$ for $i=0,1$ and all $z \in[0,1]$.

Then $X(i, j)(z)$ are continuous differentiable functions such that $0<$ $X(i, j)(z)<1$ for all $i, j$ and $z \in[0,1]$.

Proof. We prove the lemma by induction on $i$. We already know that it holds for $i=0,1$. Suppose that it holds for $0 \leq i<l$. Using the system 
(2.3) we can express $X(l, j)$ via $X(l-2, j), X(l-1, j+1)$ and $X(l-1, j-1)$ as follows:

$$
1-X(l, j)^{-1}=\frac{(1-X(l-1, j+1))(1-X(l-1, j-1)) X(l-2, j)}{X(l-2, j)-1} .
$$

We know that $X(l, j)(0)=X(i, j)_{0} \in(0,1)$, and it is clear from (4.7) that $X(l, j)$ can not be equal to 0 or 1 if all $X(l-2, j), X(l-1, j+1)$ and $X(l-1, j-1)$ lie in the interval $(0,1)$. Therefore, by our inductive assumption, $X(l, j)(z) \in(0,1)$ for all $z \in[0,1]$ and $X(l, j)(z)$ is continuous and differentiable. Hence the lemma holds for all $i \geq 0$. The case $i<0$ is treated similarly.

According to this lemma, the functions $X(i, j)=X(i, j)(z)$ that we have constructed, belong to the group $C$. We also know that the values of $X(i, j)(z)$ at 0 and 1 are equal to $X(i, j)_{0}$ and $X(i, j)_{1}$, respectively.

Note that there is a one-to-one correspondence between the coset $\mathfrak{F}_{\epsilon}$ and the set $\left\{(i, j) \in \mathfrak{S}_{\epsilon} \mid 1 \leq i \leq n+3,1 \leq j \leq n\right\}$. Hence

$$
\begin{aligned}
& \sum_{i=1}^{n+3} \sum_{j=1}^{n} X(i, j) \otimes(1-X(i, j)) \\
& =\sum_{(i, j) \in \mathfrak{F}_{0}} X(i, j) \otimes(1-X(i, j))+\sum_{(i, j) \in \mathfrak{F}_{1}} X(i, j) \otimes(1-X(i, j)) .
\end{aligned}
$$

The functions $X(i, j)=X(i, j)(z)$ satisfy the system (2.3) for all $z \in[0,1]$. By expressing $1-X(i, j)$ in terms of the $X\left(i^{\prime}, j^{\prime}\right)$ 's using formula (4.4), we obtain

$$
\sum_{(i, j) \in \mathfrak{F}_{\epsilon}} X(i, j) \otimes(1-X(i, j))=\sum_{(i, j) \in \mathfrak{F}_{\epsilon}} \sum_{\left(i^{\prime}, j^{\prime}\right) \in \mathfrak{B}_{\epsilon}(i, j)} X(i, j) \otimes X\left(i^{\prime} j^{\prime}\right) .
$$

Since the relation

$$
\left\{\left((i, j),\left(i^{\prime}, j^{\prime}\right)\right) \mid\left(i^{\prime}, j^{\prime}\right) \in \mathfrak{B}_{\epsilon}(i, j)\right\} \subset \mathfrak{F}_{\epsilon} \times \mathfrak{F}_{\epsilon}
$$

is symmetric, we conclude from formulas (4.8) and (4.9) that

$$
\sum_{i=1}^{n+3} \sum_{j=1}^{n} X(i, j) \otimes(1-X(i, j)) \in S^{2} C .
$$

Therefore, these functions satisfy the conditions of Corollary 1 . Hence the sum of the values of the Rogers dilogarithm function at $X(i, j)_{0}$ is 
equal to that at $X(i, j)_{1}$. But we can derive from the known dilogarithm identity $[8,9,10]$

$$
\sum_{j=1}^{n} L\left(\frac{\sin ^{2} \frac{\pi}{n+3}}{\sin ^{2} \frac{(j+1) \pi}{n+3}}\right)=\frac{\pi^{2}}{6} \frac{2 n}{n+3}
$$

and the Euler identity $L(z)+L(1-z)=\pi^{2} / 6$ that

$$
\sum_{i=1}^{n+3} \sum_{j=1}^{n} L\left(X(i, j)_{0}\right)=\frac{\pi^{2}}{6} n(n+1) .
$$

The identity (4.1) now follows from Corollary 1.

By using the same argument and Corollary 2 we obtain a proof of (4.2).

Finally, observe that $D(z)=0$ for all real $z$, and hence

$$
\sum_{i=1}^{n+3} \sum_{j=1}^{n} D\left(X(i, j)_{0}\right)=0 .
$$

The identity (4.3) now follows from Corollary 3.

\section{References}

1. A. Belavin, A. Polyakov, A. Zamolodchikov, Nucl. Phys. 241 (1984), 333-380.

2. A. Zamolodchikov, Adv. Stud. in Pure Math. 19 (1989), 641-674.

3. J.-L. Gervais, Phys. Lett. 160B (1985), 277-278;

R. Sasaki and I. Yamanaka, Adv. Stud. in Pure Math. 16 (1988), 271-296;

B. Kupershmidt and P. Mathieu, Phys. Lett. 227B (1989), 245-249;

T. Eguchi and S.-K. Yang, Phys. Lett. 224B (1989), 373-378;

T. Hollowood and P. Mansfield, Phys. Lett. 226B (1989), 73-79;

V. Fateev, S. Lukyanov, Kiev Preprints ITF-88-74R, ITF-88-75R, ITF-88-76R, 1988 [English translation: Sov. Sci. Rev. A Phys. 15 (1990) 1].

4. B. Feigin and E. Frenkel, Phys. Lett. B276 (1992), 79-86; Integrals of motion and quantum groups, Preprint YITP/K-1036, hep-th/9310022, to appear in Lect. Notes in Math.

5. A. B. Zamolodchikov and Al. B. Zamolodchikov, Ann. Phys. 120 (1979), 253-291.

6. T. Klassen, E. Melzer, Nucl. Phys. 338 (1990), 485-528; 350 (1991), 635-689.

7. Al. B. Zamolodchikov, Nucl. Phys. B342 (1990), 695-720; B358 (1991), 497-523; B358 (1991), 524-546; B366 (1991), 122-134.

8. L. Lewin, Polylogarithms and associated functions, North Holland, New York, 1981.

9. B. Richmond and G. Szekeres, J. Australian Math. Soc. (Series A) 31 (1981), 362373.

A. N. Kirillov and N. Reshetikhin, J. Soviet Math. 35 (1986), 2627-2643;

A. N. Kirillov, J. Soviet Math. 47 (1989), 2450-2459; Dilogarithm identities, Preprint hep-th/9408113;

W. Nahm, A. Recknagel and M. Terhoeven, Mod. Phys. Lett. A8 (1993), 1835;

E. Frenkel and A. Szenes, Duke Math. Journal 69, IMRN 2, 53-60 (1993);

J. L. Dupont and C.-H. Sah, Comm. Math. Phys. 161 (1993), 265-282;

D. Zagier, Dilogarithm identities, unpublished manuscript. 
10. E. Frenkel, A. Szenes, Journal of AMS 8 (1995), 629-664.

11. F. Gliozzi, R. Tateo, Phys. Lett. 348B (1995), 84-88.

12. Al. B. Zamolodchikov, Phys. Lett. B253 (1991), 391-394.

13. F. Ravanini, A. Valleriani and R. Tateo, Int. J. Mod. Phys. A8 (1993), 1707-1727.

14. V. V. Bazhanov and N. Yu. Reshetikhin, Int. J. Mod. Phys. B4 (1989), 115.

15. A. Klümper and P. A. Pearce, Physica A 183 (1992), 304-350.

16. A. Kuniba, T. Nakanishi, Mod. Phys. Lett. A7 (1992), 3487-3494; A. Kuniba, T. Nakanishi and J. Suzuki, Int. J. Mod. Phys. A9 (1994), 5215-5312.

17. V. V. Bazhanov, S. L. Lukyanov and A. B. Zamolodchikov, Integrable structure of conformal field theory, quantum KdV theory and thermodynamic Bethe ansatz, Preprint CLNS 94/1316, RU-94-98, hep-th/9412229.

18. R. Tateo, New functional dilogarithm identities and sine-Gordon $Y$-system, preprint DTP/95/16, hep-th/9505022.

19. S. Bloch, in Proceedings of the International Symposium on Algebraic Geometry, Kyoto 1977, Kinokuniya Book-Store Co., Ltd., Tokyo, pp. 103-114.

20. F. Gliozzi and R. Tateo, Thermodynamic Bethe ansatz and threefold configurations, Preprint DFTT-27-95, hep-th/9505102.

Department of Mathematics, Harvard University, Cambridge MA 02138

E-mail address: frenkel@math.harvard.edu

Department of Mathematics, Massachusetts Institute of Technology, CamBRIDGE MA 02139

E-mail address: szenes@math.mit.edu 